\title{
Comercio exterior e impacto ambiental
}

\section{Foreign trade and environmental impact}

1 Erika Sonia Quiñonez Alvarado Universidad de Guayaquil erika.quinoneza@ug.edu.ec

2 Pedro Alexander Avilés Almeida Universidad de Guayaquil pedro.avilesal@ug.edu.ec

3 Layla Yasmina Viteri Rade Instituto Tecnológico Universitario EuroAmericano lviteri@euroamericano.edu.ec https://orcid.org/0000-0002-3520-5183

https://orcid.org/0000-0001-5207-2645

https://orcid.org/ 0000-0002-6372-3009

Artículo de Investigación Científica y Tecnológica

Enviado: 10/12/2021

Revisado: 25/12/2021

Aceptado: 28/01/2022

Publicado:05/02/2022

DOI: https://doi.org/10.33262/ap.v4i1.1.136

Quiñonez Alvarado, E. S., Avilés Almeida, P. A., \& Viteri Rade, L. Y. (2022). Comercio exterior e impacto ambiental. AlfaPublicaciones, 4(1.1), 6-16. https://doi.org/10.33262/ap.v4i1.1.136

ALFA PUBLICACIONES, es una Revista Multidisciplinar, Trimestral, que se publicará en soporte electrónico tiene como misión contribuir a la formación de profesionales competentes con visión humanística y crítica que sean capaces de exponer sus resultados investigativos y científicos en la misma medida que se promueva mediante su intervención cambios positivos en la sociedad. https://alfapublicaciones.com

La revista es editada por la Editorial Ciencia Digital (Editorial de prestigio registrada en la Cámara Ecuatoriana de Libro con No de Afiliación 663) www.celibro.org.ec 


\section{Palabras}

claves: medio

ambiente,

industria,

comercio

internacional.

\section{Keywords:}

environment, industry, international trade.

\section{Resumen}

El siguiente trabajo está enfocado en determinar mediante la revisión bibliográfica, la relación estrecha que existe entre las actividades ligadas al comercio exterior y el deterioro que ha sufrido el medio ambiente natural. Es importante determinar que mediante el comercio internacional las naciones han llevado a cabo transacciones comerciales de sus productos con la finalidad de elevar la calidad de vida de sus ciudadanos mediante la promoción y ejecución de actividades de manufactura en las industrias de cada país, para luego ser exportados hacia otras regiones por medio del comercio internacional, el cual permite el intercambio de bienes y servicios entre regiones o países. El uso de las nuevas tecnologías ha impactado enormemente las operaciones mercantilistas a nivel mundial, y ha dado un nuevo auge y relanzamiento al comercio, pero las industrias aún siguen con una gran deuda con el medio ambiente al no cumplir con las regulaciones internacionales y locales que permitan causar el menor daño posible a la naturaleza.

\begin{abstract}
The following work is focused on determining, through a bibliographic review, the close relationship that exists between activities related to foreign trade and the deterioration suffered by the natural environment. It is important to determine that through international trade, nations have carried out commercial transactions of their products in order to raise the quality of life of their citizens through the promotion and execution of manufacturing activities in the industries of each country, to later be exported to other regions through international trade, which allows the exchange of goods and services between regions or countries. The use of new technologies has greatly impacted commercial operations worldwide and has given a new boom and relaunch to trade, but industries still have a great debt to the environment by not complying with international and local regulations that cause the least possible damage to nature.
\end{abstract}

\section{Introducción}

El medio ambiente es una de las premisas más nombradas hoy día en todos los foros mundiales, convenciones de empresarios y gobiernos, reuniones entre naciones, 
convenciones de científicos, ONG organizaciones dedicadas a la protección del planeta de talla mundial, entre otras organizaciones, con la finalidad de establecer un camino claro a seguir en cuanto a la modificación de las conductas del ser humano que han llevado a la salud del planeta a terapia intensiva, sin duda alguna no es exagerado pensar que si no se toman las medidas necesarias para tratar de revertir o al menos frenar la crisis medio ambiental la humanidad entera estaría al borde una de las más grandes crisis de extinción de la raza humana de toda la historia del planeta tierra.

El fenómeno del calentamiento global está, así, intrínsecamente ligado no solo a temas como la contaminación del aire, la salud o los recursos naturales, sino que también se encuentra interconectado con asuntos económicos y sociales, en particular, con cuestiones de equidad a escala global. Aunque quizá el vínculo no resulte evidente, numerosa literatura internacional demuestra cómo los embates del cambio climático tienen un efecto más notorio en las personas pobres (Casas, 2017). Esto resulta cierto cuando se observa los desastres que ocasionan fenómenos como huracanes más potentes, inundaciones, taludes, entre otros fenómenos que son causados por el cambio climático y afectan cada día mas a la población que cuenta con menos recursos para su subsistencia.

Esto sin duda alguna afecta directamente al desarrollo normal que deben seguir las industrias al ver como se paralizan en muchos casos la producción de las empresas, el retraso en la entrega de muchos insumos o mercancía que por situaciones climáticas no pueden ser trasladados hasta su destino final, entre otros problemas de orden ambiental que deben ser tratadas con seriedad y transparencia con la finalidad de logar un verdadero cambio en el entorno.

Por otro lado, las regulaciones en materia ambiental a nivel internacional han sido prácticamente insuficientes, ya que las grandes corporaciones en concordancia y alianza con diversos gobiernos de los países más desarrollados ponen en jaque al planeta entero al no cumplir con los protocolos establecidos por las organizaciones mundiales a las que irónicamente están suscritos, uno de los principales responsables es el sistema productivo actual sustentado en la racionalidad economicista e instrumental del capitalismo en la idea de economizar y hacer tecnológico al planeta (Zarate y Miranda, 2016).

Sin embargo, se ha visto como en la agricultura y ganadería como se afecta por desastres naturales causados por fenómenos climáticos como el niño, entre otros, lo cual sin lugar a duda genera pérdidas multimillonarias en el sector.

De esta manera, Zarate y Miranda (2016) citando a la PNUD mencionan que:

Las condiciones climáticas afectan directamente la productividad agrícola y pecuaria; la presencia de enfermedades, plagas y especies invasoras inciden sobre 
las condiciones de almacenaje, afectan el transporte y la conservación de los productos, alteran las condiciones de los mercados, afectando casi la totalidad de los procesos involucrados en las cadenas productivas y comprometiendo de esta manera la seguridad alimentaria y los medios de vida de las comunidades más vulnerables

Se intenta establecer una visión amplia de las implicancias en materia de impacto ambiental que pueden ser producidas por el comercio exterior y sus actividades asociadas.

\section{Metodología}

La investigación es de tipo documental, se realizó una revisión bibliográfica entre documentos de orden científico, revistas de investigaciones, tesis de grado, documentos de organismos internacionales emitidos bajo los más estrictos estándares científicos académicos, investigaciones científicas que proporcionan la información necesaria para realizar la documentación del tema. La investigación documental, como herramienta ayuda en la construcción del conocimiento, amplia los constructos hipotéticos de los estudiantes y como enriquece su vocabulario para interpretar su realidad desde su disciplina, constituye elemento motivador para la realización de procesos investigativos (Gómez et al., 2016).

Con este método se pretende establecer las comparativas respectivas en los temas que se tratan en la investigación

\section{Resultados y Discusión}

El impacto ambiental es un tema que ha estado presente al menos desde que la revolución industrial hizo presencia en la humanidad, desde la mecanización de los procesos en las industrias y la máquina de vapor se tornó necesaria a nivel industrial, se ha visto como la aplicación de estas nuevas tecnologías eran empleadas en los procesos y provocando impactos formidables en diversas áreas de la vida cotidiana, entre esos los que destaca es el deterioro al medio ambiente, que sin duda alguna no es el único que ha sufridos las consecuencias de la industrialización. Si bien es cierto que a los inicios de la industrialización no existía el problema climático, ahora es un problema muy presente, desde los inicios de la maquina a vapor impulsada por carbón los cambios en el entorno ambiental, como la contaminación del aire, la contaminación del agua y suelos, tala indiscriminada de árboles y selvas enteras, extinción de especies de la flora y fauna de todo el planeta. Para el siglo XVIII en donde la revolución industrial tuvo su surgimiento el comercio entre los diferentes continentes se hizo más notorio, sobre todo el realizado por medio marítimo, el comercio ultramarino exterior vinculado a la península española, por ejemplo, probablemente fue producto del crecimiento económico y demográfico de 
los distintos territorios. Se fueron agregando con el correr de las décadas nuevas rutas y mercancías y las ya preexistentes se reacomodaron a los nuevos tiempos (Camarda, 2017).

Estos cambios en el medio ambiente se fueron suscitando a medida que el comercio fue aumentando en el planeta, y es que si no existe demanda de algún bien o servicio no puede prevalecer la oferta de los mismos, es así como la industrialización provoco, entre otras cosas, que las personas de una clase menos privilegiada en ese entonces pudiesen obtener productos que anteriormente solo podían ser adquiridos por personas de altos recursos, esto debido a la poca cantidad en el mercado de determinado producto, esta situación cambia drásticamente, cuando la industrialización da paso a la producción en masa, y se llenan los anaqueles de productos, conviene recordar que la actividad humana, desde la revolución industrial y en forma creciente, ha tenido, tiene y tendrá efectos desequilibrantes en el ambiente, planteado de esta forma un problema de ética intergeneracional: Las futuras generaciones dispondrán de un planeta cada vez más desgastado Fazio (2013),por lo que el deterioro del ambiente resulta de la causa del aumento en el comercio entre países que se dio gracias a los viajes marítimos en la búsqueda de nuevas tierras, con lo que se dio el encuentro de muchas civilizaciones y en consecuencia, una transculturización que proporciono el intercambio comercial de todo tipo.

Desde ese entonces, la humanidad ha alcanzado y logrado importantes e impresionantes avances de todo tipo, sin duda alguna se han dado respuesta a muchas necesidades de la humanidad, sobre todo en medicina, y en desarrollo educacional, sin embargo, estos avances han tenido un alto costo para el medio ambiente, y sin duda alguna han desencadenado desequilibrios de gran envergadura a la tierra y sus elementos. En este contexto resulta importante establecer la terminología que se asocia a los delitos o daños al ambiente, según Chirino et al. (2016) es importante señalar que el daño ambiental es la afectación de la calidad y cantidad de los componentes ambientales debido al accionar humano, generando efectos nocivos al medio como la contaminación ambiental, por lo cual para el caso del resultado directo a la afectación ambiental viene dado por dos principales actores, uno el daño ambiental el cual es el resultante de algún tipo de actividad humana la cual da como daño colateral, en muchos casos los efectos nocivos al ambiente, mientras que para Martínez \& Schlupmann (1993, citado por Chirino et al. (2016), los delitos ambientales es toda acción humana que va contra el ambiente imputable a un sujeto a quien se sanciona por una pena legal, en este caso se presenta un delito el cual estaría tipificado en las leyes de cada nación, y acarrea una pena o sanción.

Por otro lado, las implicancias morales y éticas en el tema ambiental están ligadas al desarrollo económico de ciertas naciones, es decir, no es un secreto para nadie que luego de que las industrias comenzaran sus operaciones en prácticamente todo rincón del 
planeta, estas generaron no solo productos terminados, sino que por otro lado se generó uno o varios subproductos que en muchos o más bien demasiados casos resultaron ser nocivos para el medio ambiente, no es sino que mencionar el caso de las empresas que emiten gases de efecto invernadero y han provocado el aumento de la temperatura del planeta, así como la emisión de aguas residuales y residuos químicos los cuales son desechados en ríos y mares contaminando así grandes extensiones de aguas limpias y deteriorando la calidad de los suelos, sin dejar de lado los efectos que a la salud que en los seres humanos han provocado múltiples consecuencias negativas, como por ejemplo el uso indiscriminado de fertilizantes y pesticidas para los cultivos y han "envenenado" a muchos trabajadores de la agricultura a nivel mundial, esto sin duda son ejemplos muy cortos sobre la problemática real y compleja que envuelve el tema del daño al ambiente y los diferentes actores que conviven en él.

Ahora bien, como se mencionó anteriormente, el impacto ambiental comenzó con mayor énfasis a mediados del siglo XVIII, con la llegada de la revolución industrial, y a causa de eso las nuevas rutas transoceánicas, nuevas rutas por tierra, y muchos más tarde por aire, se acoplarían para formar la gran y diversa red de comercio internacional que hoy se conoce, con la dinámica propia de un mercado en donde la oferta y la demanda está dada por la capacidad de exportación de cada país y la demanda de cada país en torno a la adquisición de determinados productos, es un juego diverso que proporciona múltiples ventajas, al menos desde el punto de vista mercantilista, ya que muchos países, sobre todo los países del primer mundo, tienen todas las posibilidades de comercializar sus productos y entrar al juego del comercio internacional, sin embargo cabe mencionar que los países menos privilegiados a nivel económico les resulta mucho más difícil poder ser parte de este juego, ya que las pautas que dicta el comercio son múltiples pero estrictas. Sin embargo, hoy día cada región se encuentra de alguna u otra manera dentro de la balanza del comercio internacional, ya que si no exporta debe importar, ya que todas las economías del mundo, exceptuando algunos países, están en la obligación de adquirir productos de algún otro país o región, o viceversa, ya que se ha visto que ningún país puede subsistir y sostenerse en una economía globalizada, la cual obliga a los mercados internacionales a tener una actividad muy dinámica y en estos tiempos muy cambiante, sobre todo con la incursión de nuevas y mejores tecnologías que aportan un avance importante en la forma y manera en que se comercializa.

De acuerdo con lo anterior, se puede mencionar que las actividades humanas han sido el detonante para que el ambiente haya cambiado de tal manera que hoy se encuentra a la humanidad diezmada por el cambio climático, el aumento demográfico y la escasez de recursos por la degradación ambiental han hecho mella en las actividades normales del ser humano, en este contexto citado por (Valencia, 2018): 
La degradación del medio ambiente aumenta a medida que el nivel de ingreso lo hace; esto sucede porque la economía está basada en actividades primarias (agricultura y minería, principalmente). Después, cuando la economía llega a una fase industrial, la degradación es mayor y el nivel de ingresos aumenta más, esto hasta el punto de inflexión de la curva, en el cual la economía desarrolla cada vez más actividades en servicios e industria con procesos de manejo de residuos, con base en nuevas tecnologías, manteniendo el crecimiento en el ingreso, pero con una constante caída en la degradación ambiental.

Esto sin duda representa una interesante hipótesis en la cual el desarrollo industrial daría paso a una mejora sustancial en cuanto a la disminución del deterioro ambiental, o al menos se mantendría una constante en dicha degradación, sin embargo la realidad es otra, en contraste con lo propuesto en la hipótesis anterior, el impacto negativo hacia el medio ambiente a causa de la actividad industrial en el mundo es notorio, en cifras se puede mencionar según Ovalle (2018) menciona datos interesantes sobre el deterioro medioambiental, entre los que destaca:

- China en la actualidad es el mayor emisor de gases $\mathrm{CO} 2$ del planeta, sin embargo, históricamente desde 1850- PNUD ha sido Estados Unidos el país responsable de la emisión de gases invernadero del planeta tierra.

- La temperatura Global aumento $1^{\circ}$ entre 1880 y 2015, lo cual acarrea problemas severos en el medio ambiente.

Entre muchos otros problemas que lamentablemente son causantes de millones de muertes directas e indirectamente a causa de los embates del desequilibrio ecológico mundial.

Se debe mencionar, con especial ahínco, que la actividad industrial guarda una estrecha relación con el deterioro medio ambiental, según él Banco Mundial (2021) la industria en la causante directa de:

La gestión inadecuada del medioambiente y de los recursos naturales da lugar a pérdidas económicas considerables; por ejemplo, un monto estimado de USD 80.000 millones al año se desaprovechan, debido a la mala gestión de la pesca en los océanos. La contaminación atmosférica es el principal riesgo sanitario y los costos para el mundo se estimaron en USD 8,1 billones en 2019, cifra que equivale al $6,1 \%$ del PIB mundial.

Esto deja en manifiesto que el derroche y mal uso de los recursos naturales, solo trae como consecuencia el desequilibrio no solo de la flora y la fauna, ni tampoco el cambio del clima sino que genera un efecto domino en el que las finanzas a nivel mundial se 
tambalean, se quiebran y en el mayor de los casos declinan, ya que los mercados mundiales dependen de las condiciones medioambientales para que las industrias subsistan, puedan tomar de la tierra los recursos y ser transformados en los diferentes productos que se comercializan, incluso la economía mundial es tan frágil que depende hasta de las condiciones climáticas para poder transportar los bienes a comercializar.

Por otro lado, se encuentra el ser humano como pieza fundamental del normal desarrollo del comercio internacional, ya que la mano de obra humana aun ocupa el mayor activo para la materialización de la manufactura de toda empresa, aun y cuando en muchas industrias la mano de obra mecanizada ha reemplazado muchos puestos de trabajo, el ser humano se hace indispensable para la producción en la industria, es por esto que se hace pertinente la afirmación que realiza el Banco Mundial (2021) en su artículo, cuando menciona que:

La pandemia de COVID-19 pone de manifiesto los vínculos que existen entre la salud humana y la naturaleza. Varios estudios han revelado la conexión entre la destrucción del hábitat natural y el aumento del riesgo de enfermedades zoonóticas. Los patógenos prosperan donde hay cambios medioambientales, por ejemplo, la deforestación, y cuando los ecosistemas naturales se encuentran sometidos a estrés a raíz de la actividad humana y el cambio climático.

Sin lugar a dudas la protección del medio ambiente natural es un asunto de vital importancia para la continuidad de la especie humana y las actividades a nivel industrial, la incorporación de las nuevas tecnologías para resolver diferentes problemas de logística en el comercio exterior son la boga de la actualidad, y es que se puede contar con estrategias de transporte como la Logística Electrónica o E-logistic, la cual explica Córdova (2018) el proceso de la logística es lograr que los productos y servicios lleguen en el momento preciso y en las condiciones adecuadas, al hablar de la E-Logistic lo referimos que son procesos realizados por medio del Internet, haciendo uso de la red de comercialización más ampliamente usada en la actualidad, muchos de los comercios mantienen su plataforma digital mediante la cual proporcionan información sobre sus productos, estos productos son transportados por operadores logísticos que actualmente están implementando una visión de trabajo verde en la que sus labores no impacten negativamente al medio ambiente.

Tal es el caso de las llamadas logística verde, la cual está basada en la mejora del uso de los materiales logísticos, buscando impulsar un desarrollo de la economía concentrada en materias primas, almacenamiento, procesos y transporte amigables con el medio ambiente; que, combinados con las tácticas de clientes, empresas y estados, forman iniciativas para su implementación y un desarrollo sostenible (Cordova, 2018). 
Otro caso que se está presentando en la comercialización internacional es la llegada de la cuarta revolución industrial, esta no se define por un conjunto de tecnologías emergentes en sí mismas, sino por la transición hacia nuevos sistemas que están construidos sobre la infraestructura de la revolución digital (anterior) (Perasso, 2016). Este nuevo enfoque en el que mencionan las interconexiones entre elementos ciber físicos los cuales son capaces de comunicarse entre sí y sin la interacción o la necesidad de la intervención del ser humano, buscan sustituir la mano de obra humana, y de alguna manera intenta reducir al mínimo los residuos que genera la empresa tradicional e impacta el medio ambiente. Esto viene a revolucionar aún más el ya avanzado mundo del comercio exterior en el que se está depositando toda la responsabilidad de la cadena de valor y de suministros en manos de sistemas cibernéticos totalmente autónomos, estas tecnologías han proporcionado una ventana nueva a la manera de cómo gestionar la comercialización de bienes y servicios en el comercio internacional, ya que los consumidores apuntan cada día más hacia el menor tiempo posible en que llegue su pedido, hoy en día la automatización de la mayoría de los procesos del comercio han hecho posible que mejore el comercio internacional, sin embargo hay muchas cuentas pendientes con la necesidad de que las empresas tomen en consideración la protección del medio ambiente sin necesidad de disminuir sus ganancias y beneficios en los temas relacionados al comercio exterior.

\section{Conclusiones}

- El cambio climático no está desligado de las actividades industrializadas, la evolución de los medios de producción en el mundo ha representado un importante avance para la mejora de la calidad de vida del ser humano en general, históricamente el hombre ha necesitado suplir ciertas necesidades básicas que han escalado hasta llegar a lo que hoy se conoce como una economía consumista, esto ha provocado que las industrias tengan la necesidad de producir mucho más y en menor tiempo, los mercados internacionales ahora se enfocan en lograr posicionar más y mejores productos en el menor de los tiempos posibles, con la finalidad de lograr mejores ganancias.

- Esto ha traído como consecuencia el uso desmedido y sin control de los recursos naturales del planeta, los procesos que transforman estas materias primas generan miles de toneladas de desechos tóxicos al planeta ocasionando así la mayor crisis ecológica en la historia del planeta tierra, el comercio es sin duda uno de los grandes culpables de este desequilibrio en el que los seres humanos son los afectados directos ya que miles de personas mueren a diario a causa de algún desastre natural ocasionado por el cambio climático. 


\section{Referencias Bibliográficas}

Banco Mundial. (01 de octubre de 2021). Medio Ambiente. Panorama General: https://www.bancomundial.org/es/topic/environment/overview\#1

Camarda, M. (2017). Una Aproximación al Comercio Exterior Ultramarino de la Monarquía Española a Fines del Siglo XVIII. Magallánica, Revista de Historia Moderna.

Casas, M. (2017). Comisión Económica para América Latina y el Caribe. https://repositorio.cepal.org/bitstream/handle/11362/41101/S1700115_es.pdf?se quence $=1 \&$ is Allowed $=\mathrm{y}$

Chirino, Y., López, E., \& Peñaloza, A. (2016). Daños y delitos ambientales como conceptos discernibles en la enseñanza de la Química del Instituto Pedagógico de Caracas. Estudio preliminar desde la perspectiva estudiantil. Revista de Investigación.

Córdova, A. (2018). Impacto de la Logística Electrónica (e-logistics) en el Medio Ambiente en las Pymes de Servicios del Comercio Exterior. Guayaquil: Tesis de Grado para Optar por el Titulo de Ingeniero en Comercio Exterior.

Fazio, H. (2013). Economía, Ética y Ambiente: En Un Mundo Finito. Buenos Aires: Universitaria de Buenos Aires.

Gómez, D., Carranza, Y., \& Ramos, C. (2016). Revisión Documental, Una Herramienta para el Mejoramiento de las Competencias de Lectura y Escritura en Estudiantes Universitarios. UNACH Revista Chakinan, 45-56.

Ovalle, M. (2018). 14 impactantes Cifras Sobre el Deterioro del Medio Ambiente. Ladera Sur.

Perasso, V. (12 de octubre de 2016). Qué es la cuarta revolución industrial. https://docs.ufpr.br/ jrgarcia/macroeconomia_ecologica/macroeconomia_ecolog ica/Qu\%C3\%A9\%20es\%20la\%20cuarta\%20revoluci\%C3\%B3n\%20industrial.p df

Valencia, C. (2018). Fauna Silvestre en Colombia: Entre la Ilegalidad y las Oportunidades del Comercio Internacional en las CITES. Revista Virtual Fauna Silvestre, 128145. 
Zarate, A., \& Miranda, G. (2016). Impacto del Cambio Climático en la Seguridad Alimentaria en Zonas Campesinas Vulnerables de los Andes del Perú. Revista Mexicana de Ciencias Agrícolas.

El artículo que se publica es de exclusiva responsabilidad de los autores y no necesariamente reflejan el pensamiento de la Revista Alfa Publicaciones.

\section{Liencia}

El artículo queda en propiedad de la revista y, por tanto, su publicación parcial y/o total en otro medio tiene que ser autorizado por el director de la Revista Alfa Publicaciones.
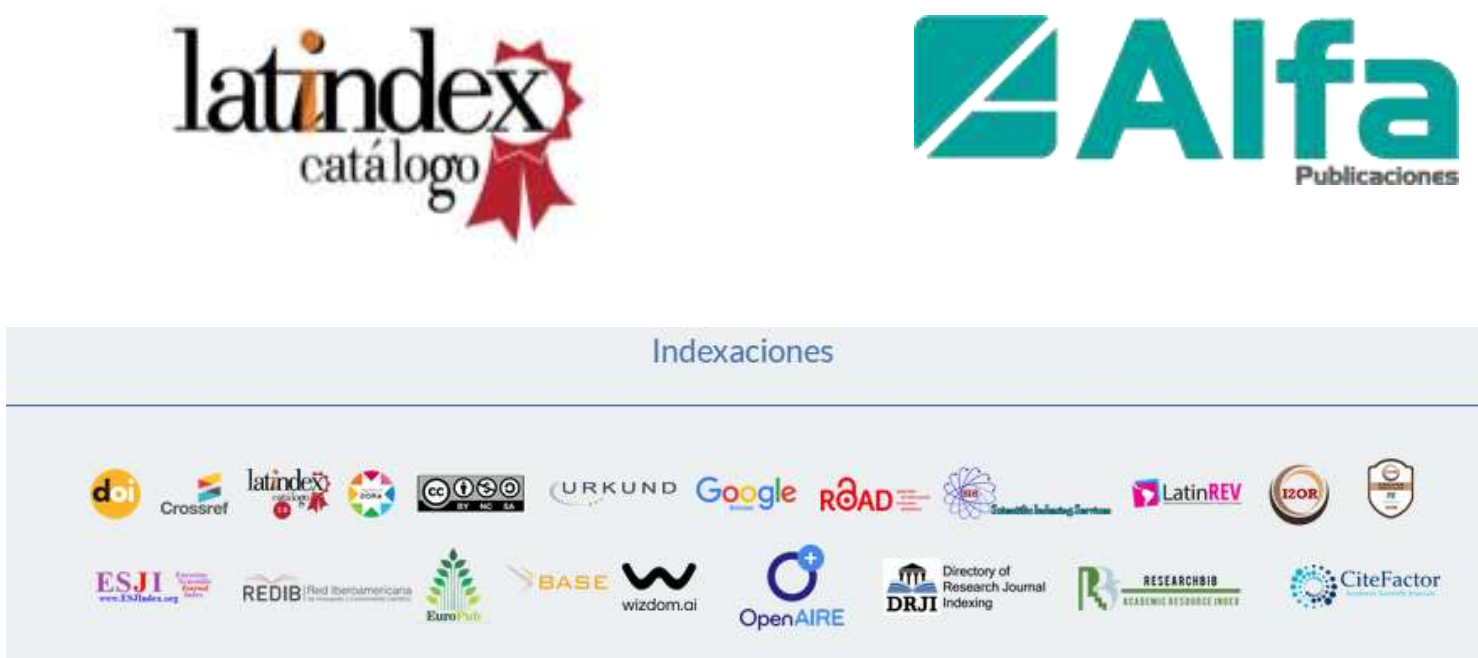